\title{
DERECHO Y POLÍTICAS AMBIENTALES DE LAS ISLAS CANARIAS
}

\author{
ADOLFO JIMÉNEZ JAÉN \\ Profesor Titular de Escuela Universitaria \\ Universidad de Las Palmas de Gran Canaria
}


Sumario: 1. Plan Especial de Gestión del Riesgo de Inundación de la Demarcación Hidrográfica de La Gomera. 1.1. Introducción.

\section{PLAN ESPECIAL DE GESTIÓN DEL RIESGO DE INUNDACIÓN DE LA DEMARCACIÓN HIDROGRÁFICA DE LA GOMERA}

\subsection{Introducción}

Mediante el Decreto 10/2020, de 20 de febrero (BOC 77, de 9 de marzo de 2020) se ha aprobado el Plan Especial de Gestión del Riesgo de Inundación de la Demarcación Hidrográfica de La Gomera. Este Plan viene a complementar el Plan Hidrológico de la Demarcación Hidrológica de La Gomera, aprobado mediante el Decreto 137/2018, de 17 de septiembre, uno de cuyos contenidos es precisamente la protección contra inundaciones (véase el artículo 42 de su Normativa, que se remite al plan de gestión de riesgos de inundación).

No obstante, ha de tenerse en cuenta que el marco normativo de este plan es más amplio. Así hay que hacer referencia, en primer término, en el ámbito europeo, la Directiva 2000/60/CE, del Parlamento Europeo y del Consejo, de 23 de octubre de 2000 incorporada a nuestro ordenamiento jurídico mediante el artículo 129 de la Ley 62/2003, de 30 de diciembre de Medidas Fiscales, Administrativas y del Orden Social incluyó entre sus objetivos que el marco para la proyección de las aguas debe contribuir a paliar los efectos de las inundaciones y sequías. De manera más específica, referido ya a las inundaciones, ha de citarse la Directiva 2007/60/CE, del Parlamento Europeo y del Consejo, de 23 de octubre de 2007, relativa a la evaluación y gestión de los riesgos de inundación, cuya transposición al ordenamiento jurídico español se realizó a través del Real Decreto 903/2010, de 9 de julio, de evaluación y gestión de riesgos de inundación.

La Directiva de Inundaciones y en el Real Decreto 903/2010, de 9 de julio, establece que la planificación de los riesgos de inundaciones comprende tres fases: 1. La evaluación preliminar del riesgo de inundación. 2. La elaboración de mapas de peligrosidad y de riesgo de inundación. 3. La aprobación de los planes de gestión del riesgo de inundación. 
De acuerdo con la Ley de Aguas de Canarias este tipo de planes se encuadran dentro de los Planes Hidrológicos Especiales que son aquellos que se circunscriben a extremos concretos contemplados en el Plan Hidrológico Insular, al cual, por tanto, complementan.

En este sentido, y tal y como establece la memoria del propio Plan, el Plan de Gestión de Riesgo de Inundación tiene una doble naturaleza jurídica: sectorial y territorial.

Por un lado, la naturaleza sectorial viene dada por la Directiva 2007/60/CE, del Parlamento Europeo y del Consejo, de 23 de octubre de 2007. Ahora bien, el propio plan establece que teniendo en cuenta las particularidades del sistema de planeamiento de Canarias, un enfoque únicamente sectorial resulta insuficiente para dar cumplimiento al contenido de los PGRI definido por el anexo del Real Decreto 903/2010, de 9 de julio.

En este sentido se señala que las medidas de ordenación territorial y urbanismo que incluye el Plan no pueden tramitarse dentro de un plan de esta naturaleza. En este sentido hay que recordar que el artículo 15.1 del mencionado Real Decreto, establece que "Los instrumentos de ordenación territorial y urbanística, en la ordenación que hagan de los usos del suelo, no podrán incluir determinaciones que no sean compatibles con el contenido de los planes de gestión del riesgo de inundación, y reconocerán el carácter rural de los suelos en los que concurran dichos riesgos de inundación o de otros accidentes graves". Pues bien, es el propio Plan el, que ya indica la clasificación como suelo rústico de las zonas inundables.

Es decir, se podrían desarrollar una serie de medidas genéricas desde el punto de vista territorial, no así desde su contenido, para que el planeamiento urbanístico y/o territorial las utilizase, incluyendo las que consistan en limitaciones al régimen de usos del suelo, es decir no sería el PGRI el que limitase el régimen del suelo directamente, sino que establecería una "metodología", que los planes generales y territoriales utilizarían para ello.

Por ello, la Memoria opta por una doble naturaleza jurídica que combina las características de un plan sectorial y las de un plan territorial, lo que condiciona tanto el contenido del documento como el proceso de elaboración y aprobación 
del Plan. En este sentido se concluye que "En conclusión, el PGRI se incorpora al Sistema de Planeamiento de Canarias, como Plan Territorial, eso sí con una fuerte carga sectorial, circunscribiéndose ese carácter territorial a la implementación de una serie de medidas genéricas respecto al régimen del suelo que podría estar afectado por el riesgo de inundación, que han de ser desarrolladas por el planeamiento urbanístico y/o territorial, incluyendo en ese desarrollo las que consistan en limitaciones al régimen de usos del suelo o impongan condicionantes a los usos y/o tipologías".

Por último, debe precisarse que, desde el punto de vista territorial y urbanístico, los Planes Especiales de Gestión del Riesgo de Inundación merecen la calificación jurídica de "planes sectoriales con impacto sobre el territorio", de acuerdo con la Disposición adicional cuarta de la Ley 4/2017, de 13 de julio, del Suelo y de los Espacios Naturales Protegidos de Canarias (en particular, el apartado 3 de dicha disposición).

Estamos, por tanto, ante un plan que, una vez entre en vigor, tendrá la consideración de Plan Territorial Especial y que, además, prevalecerá sobre los restantes instrumentos de ordenación territorial, ambiental y urbanística vigentes; todo ello de conformidad con el apartado 2 de esa Disposición adicional, en relación con el artículo 15.1 del Real Decreto 903/2010, de 9 de julio, de evaluación y gestión de riesgos de inundación.

Este Decreto entrará en vigor el día 11 de marzo de 2020.

El contenido del Plan está contemplado en su Anexo.

\subsection{Causas del riesgo de inundaciones}

Según la memoria del Plan, "Relacionado con el cambio climático, en la Evaluación Preliminar del Riesgo de Inundaciones de la demarcación hidrográfica de La Gomera se extrajo la conclusión de que la gran incertidumbre de los resultados obtenidos en diversos estudios no permitía cuantificar actualmente la alteración que el cambio climático podía suponer a nivel de fenómenos extremos de precipitación. 
En La Gomera se han padecido durante los últimos años los efectos de varias borrascas significativas, cuyas descargas de intensas lluvias han generado daños importantes en diversas zonas de la isla.

Sin embargo, la información disponible y los indicios que se tienen hasta el momento apuntan a un incremento de la frecuencia de fenómenos meteorológicos extremos, no tanto de su magnitud".

A continuación, la memoria hace referencia a diversos estudios sobre el cambio climático, concretamente son los siguientes:

a) VI Documento Técnico del IPCC y Quinto informe de evaluación (AR5).

b) Agencia Estatal de Meteorología (AEMET).

c) Libro Blanco del Agua.

d) Centro de Estudios y Experimentación de Obras Públicas (CEDEX).

a. CEDEX 2010

b. CEDEX 2017

e) Estrategia canaria de lucha contra el cambio climático

f) Proyecto Climatique

g) Proyecto Clima-Impacto

Señalaremos a continuación algunas de las conclusiones de estos trabajos, de acuerdo con lo que se establece en la memoria del Plan.

Así, respecto del IPCC se señala que "Las previsiones del VI Documento Técnico del IPCC (Bates et al, 2008) en latitudes medias similares a la que ocupa España indican como probable el aumento de la frecuencia e intensidad de los episodios de precipitación, así como una disminución de valores medios en verano. En el Quinto Informe de Evaluación (AR5) del IPCC (201cualquier punto, en relación con fenómenos observados, que "es probable que la frecuencia o intensidad de las precipitaciones intensas haya aumentado en Europa" y, con relación a cambios futuros, que "los eventos de precipitación extrema sobre la mayoría de las tierras de latitudes medias y regiones tropicales húmedas serán muy probablemente más intensos y frecuentes".

Respecto de la Agencia Estatal de Meteorología, se indica que "En el documento "Generación de escenarios regionalizados de cambio climático en España" 
confeccionado por la AEMET en 2008, se indica que la tendencia histórica de la precipitación no ha mostrado un comportamiento tan definido como la temperatura, aunque los modelos aplicados parecen revelar un descenso paulatino de la precipitación a lo largo de este siglo".

Para las Islas Canarias se ha utilizado la técnica de regionalización estadística que relaciona los datos a gran escala de los modelos climáticos globales con datos a escala local o regional, mediante la aplicación de dos tipos de algoritmos empíricos basados en las técnicas de análogos y de regresión lineal.

De entre los parámetros proyectos se ha escogido: precipitación, precipitaciones intensas, duración de periodos secos, y número de días de lluvia.

En la Memoria se indica que en el año 2020 se aprecia que los cambios de duración del periodo seco permanecen más o menos estables, no obstante, si se tiene en cuenta el número de días de lluvia en La Gomera se puede observar que la tendencia es decreciente ya desde el año 2020.

Por su parte, el Libro Blanco del Agua en España expone que el cambio climático produciría, en el escenario menos pesimista, una disminución del $5 \%$ en las aportaciones totales en régimen natural en España, siendo el impacto más severo en determinadas regiones, entre ellas las Islas Canarias. Esta disminución vendría acompañada de una mayor variabilidad anual, interanual y estacional.

En el informe de 2010 se señala que las proyecciones pronostican una reducción generalizada de la precipitación conforme avanza el siglo XXI, El conjunto de proyecciones en el escenario de emisiones más desfavorable supone decrementos de precipitación media en España en el entorno del -5\%, -9\% y $17 \%$ durante los periodos 2011-2040, 2041-2070 y 2071-2100 respectivamente. La región insular de Canarias es una de las más afectadas por esta disminución de la precipitación, alcanzando valores de reducción de hasta el $20 \%$ en el periodo 2011-2040.

El estudio de 2017 emplea nuevas proyecciones climáticas resultantes de la utilización de modelos climáticos más completos que los modelos acoplados atmósfera-océano empleados en el informe previo elaborado por el CEDEX en 
2010 titulado "Estudio de los impactos del cambio climático en los recursos hídricos y las masas de agua". Mientras que en el informe anterior del año 2010 se hizo uso de los modelos y escenarios disponibles para el Informe AR4 del IPCC, en este informe de 2017 se han utilizado los del AR5.

Por otro lado, también se pronostica un cambio en el régimen de sequías para cada periodo de impacto futuro con relación al periodo de control. La mayoría de las proyecciones climáticas muestran un futuro en el que las sequías serían más frecuentes, acusándose ese efecto cuanto más nos alejamos en el siglo XXI. No obstante, hay proyecciones que no muestran tan clara esa señal, especialmente en cuencas del Levante y Canarias.

Para el periodo 2040-2070 hay una reducción de Precipitaciones según casi todas las proyecciones, En general, hay un similar patrón de reducción, más intenso hacia el SO peninsular y en Canarias y menor reducción, o incluso aumento de PRE en algunas zonas del este peninsular. Por el contrario, U4A y U8A muestran un patrón contrario, con mayores descensos hacia el este.

Por su parte, la Estrategia canaria de lucha contra el cambio climático. Tal y como se indica en el Plan Hidrológico, el marco de referencia para la adaptación del cambio climático en Canarias se establece por medio de la Estrategia Canaria de Lucha contra el Cambio Climático, aprobado por el Consejo de Gobierno el 17 de marzo de 2009.

El proyecto tiene tres grandes objetivos: la lucha contra los efectos adversos, actuar contra los efectos de este fenómeno y aprovechar los posibles beneficios que genera el Cambio Climático, como puede ser la introducción de nuevas especies.

En el archipiélago canario el organismo responsable de la coordinación de las medidas propuestas en dicha estrategia es la Viceconsejería de Medio Ambiente.

El documento analiza de forma pormenorizada diferentes aspectos del ámbito terrestre, marino y de los sectores horizontales que se ven afectados por el cambio climático y a los cuales realiza una serie de recomendaciones para paliarlo. Entre los aspectos puestos de relieve en el documento figuran las 
infraestructuras preparadas para la llegada de ciclones y tormentas a las islas y los riesgos en la salud de las personas del incremento de las olas de calor o de temperaturas máximas con viento sahariano. Además, se analiza en el plan la subida del nivel del mar.

En cuanto al Proyecto Clima-Impacto, en el año 2013 el Gobierno de Canarias puso en marcha el Proyecto Clima-Impacto, con el que se pretende mejorar el conocimiento sobre los efectos del calentamiento global y su incidencia en la Macaronesia. Comprende acciones de análisis, monitorización y divulgación, orientadas en última instancia a sensibilizar a la población sobre las consecuencias del cambio climático.

El proyecto centra su actuación en la Región Canaria, aunque también a un país tercero de su entorno geográfico y cultural como es el Archipiélago de Cabo Verde. Se organiza en cuatro pilares básicos:

- Repositorio de documental.

- Efectos del cambio climático y vulnerabilidades.

- Seguimiento del cambio climático.

- Formación y divulgación.

Uno de los objetivos del Proyecto Clima-Impacto es evaluar los efectos del calentamiento a nivel local y determinar las zonas más vulnerables.

El procedimiento de trabajo consta de las siguientes fases:

1. Análisis de datos climáticos de estaciones meteorológicas (temperatura y precipitación).

2. Corrección de errores y mejora de la calidad de los datos (gaps, outliers y homogenización).

3. Cálculo de tendencias en la evolución en el tiempo de la temperatura y la precipitación.

4. Obtención de un modelo climático probabilístico de cada isla con registros de los cambios de temperatura y precipitación por sectores geográficos.

5. Definición de escenarios climáticos.

6. Interpolación de los datos de las estaciones al conjunto de la isla, para los escenarios climáticos definidos. 
7. Obtención de un atlas climático.

8. Modelizar la distribución de los bosques, y especies y cultivos indicadores.

9. Determinación de impactos sobre la biodiversidad (especies y bosques) y la agricultura.

10. Evaluación del reparto geográfico de vulnerabilidades (islas, municipios y áreas protegidas).

La lucha contra el cambio climático exige una monitorización precisa de sus efectos y consecuencias, así como de las medidas que se adoptan para combatirlo. Las acciones de mitigación y de adaptación tienen aquí un papel crucial.

Todas estas etapas exigen cálculos complejos que incluyen la elaboración de programas informáticos y manejo de software de modelización y sistemas de información geográfica. Intervienen en distintos momentos instituciones gestoras y científicas, empresas consultora y representantes de la sociedad civil.

\subsection{Determinación de las zonas inundables}

Una vez analizada la información, se procede a determinar las áreas de riesgo potencial significativo (ARPSIs, según sus siglas en español, o ASPFRs, según sus siglas en inglés) que son aquellas zonas en las que se ha llegado a la conclusión de que existe un riesgo potencial de inundación significativo o en las cuales la materialización de tal riesgo pueda considerarse probable, según el art. 5 de la Directiva.

De acuerdo con la Memoria, el proceso sigue la siguiente secuencia. En primer lugar, se procedió a identificar los registros de riesgos potenciales, esto es, reconocer la posibilidad de la existencia de un riesgo por avenidas en distintos lugares y en función de diferentes causas. Igualmente, se procedió a determinar los registros de riesgos significativos, es decir, aquellos registros potenciales cuyos riesgos pudieran ser significativos. Finalmente, se ha procedido a la constatación de los riesgos significativos anteriormente determinados.

Analizada la información disponible en el Inventario de Riesgos Hidráulicos, se considera que las zonas anegables próximas a los cauces son, por lo general, 
escasas y su alcance se encuentra bastante limitado, ello como consecuencia del abrupto relieve y pronunciada pendiente del territorio.

Se han identificado todo tipo de problemas como los relacionados con la ocupación urbana, viaria, o agrícola del cauce u otro tipo de riesgos derivados de insuficiencia de las obras de cruce con carreteras. Asimismo, destaca la importancia del fenómeno torrencial, que se pone de manifiesto por otra casuística diferente, como es la concentración de acarreos sólidos y la generación de escorrentías de ladera.

a) Metodología General para la determinación de las ARPSIS costeras

Consultado el Catálogo Nacional de Inundaciones Históricas se ha llegado a la conclusión de que la información sobre inundaciones en zonas costeras de origen marítimo, es decir, producidas por niveles extremadamente altos de la superficie del mar, es prácticamente inexistente.

Por otra parte, la aplicación de métodos geomorfológicos para identificar indicios de inundaciones pasadas en zonas costeras resulta poco eficaz porque, si la costa es elevada, no se han producido inundaciones, y si la costa es baja, el intenso desarrollo urbanístico y las numerosas regeneraciones de playas que se han llevado a cabo durante las últimas décadas ha hecho que, por un lado, hayan desaparecido dichos indicios y, por otro, que la topografía y las características hidráulicas (permeabilidad, rugosidad, pendiente) del terreno se hayan modificado sustancialmente.

En consecuencia, la evaluación preliminar de riesgos de inundación en las zonas costeras de esta Demarcación Hidrográfica se ha hecho fundamentalmente comparando los niveles del mar excepcionalmente elevados (período de retorno de 500 años) con las cotas actuales del terreno.

b) Delimitación de las zonas inundables

Se han delimitado zonas inundables por marea y zonas inundables por oleaje siguiendo dos procedimientos diferentes.

El primero de ellos, que denominaremos inundabilidad por mareas, corresponde a las causas de sobreelevación que permanecen durante horas o días, tiempo suficiente para que el nivel del mar se propague hasta cualquier punto cuya cota 
se encuentre por debajo de dicho nivel. Entre éstas se consideran naturalmente las mareas astronómica y meteorológica, pero también se ha considerado como tal el valor del remonte medio, porque es un nivel que se sobrepasa aproximadamente la mitad del tiempo que dura un temporal. En consecuencia, se ha considerado que es inundable cualquier punto cuya cota sea inferior a la suma de la cota del nivel de marea de una pleamar media más el remonte medio calculado para un período de retorno de 500 años, siempre que exista una conexión hidráulica con la costa o que pueda formarse en condiciones de sobreelevación extraordinaria. El valor del remonte medio que se utiliza en cada tramo de costa es el que corresponde a la orientación de éste.

El segundo criterio, que denominaremos inundabilidad por oleaje, corresponde al efecto del remonte de las olas. Desde este punto de vista se ha considerado inundable cualquier punto cuya cota sea inferior a la máxima cota que puede alcanzar el nivel del mar por efecto combinado de marea y oleaje con un período de retorno de 500 años, siempre que se encuentre a una distancia de la costa inferior a un valor proporcional a dicha cota.

En la metodología diseñada, los valores de cotas de inundación a aplicar a cada tramo de costa dependen de la orientación de estas. No obstante, se comienza haciendo un cálculo preliminar de las zonas inundables por marea y por oleaje utilizando como cotas las correspondientes a la orientación pésima para esa Demarcación. Para los tramos de costa en los que el ancho de la zona inundable sea inferior a $50 \mathrm{~m}$ los resultados se han considerado definitivos.

C) Criterios y objetivos ambientales especificados en el Plan hidrológico relacionados con las ARPSIs

De manera general, los objetivos medioambientales (artículo 92 bis Texto Refundido de la Ley de Aguas y artículo 35 del Reglamento de Planificación Hidrológica) pueden agruparse en las categorías que se relacionan en la siguiente figura:

A su vez, los criterios para la clasificación y evaluación del estado de las masas de agua superficiales y subterráneas se definen en la Sección 5, del Reglamento de Planificación Hidrológica. 
A continuación, se recoge un primer análisis del estado de las masas de agua y los objetivos ambientales correspondientes a las Áreas con Riesgo Potencial Significativo por Inundación (ARPSIs) así como el estado y los objetivos ambientales de las masas de agua subterráneas.

Es importante destacar que 3 ARPSIs no son masas de agua de la DMA, por lo que de ellas no se dispone de datos de estado ni de objetivos medio ambientales. Con estos datos ambientales se extraen las siguientes conclusiones, por tipo de ARPSI:

ARPSIs fluviales superficiales y de transición. A esta categoría pertenecen el $42,86 \%$ de las ARPSIs (son 3 de los 7 ARPSIs establecidas), y ninguna es masa de agua.

ARPSIs costeras. Como se ha visto con anterioridad la Dirección General de Sostenibilidad de la Costa y del Mar del MAPAMA, ha identificado las zonas clasificadas como de riesgo alto significativo de inundación por origen marino en el ámbito de la Demarcación Hidrográfica de La Gomera. En la EPRI se establecieron finalmente 4 ARPSIs costeras.

Las ARPSIs costeras representan el 57,14\% del total de las 7 ARPSIs. En cuanto a la naturaleza, estado y objetivos ambientales de estas masas de agua, éstas son las conclusiones:

- De las 4 ARPSIs costeras, todas se encuentran en estado Natural.

- Respecto al estado global y los objetivos, se ha de destacar que todas las ARPSIs costeras se encuentran en un estado Bueno o muy bueno.

Estado de las masas de agua subterráneas

A la hora de establecer el estado y los objetivos ambientales de las masas de agua subterráneas, se acude también a la información contenida en el Plan hidrológico de la demarcación. Los objetivos para las aguas subterráneas son los siguientes:

- Evitar o limitar la entrada de contaminantes y su deterioro.

- Proteger, mejorar y regenerar las masas de agua subterránea y garantizar el equilibrio entre la extracción y la recarga 
- Invertir las tendencias significativas y sostenidas en el aumento de la concentración de cualquier contaminante derivado de la actividad humana, reducir la contaminación.

Analizando la información se observa que, de las 3 masas de agua subterráneas, todas se encuentran en Buen estado cuantitativo y químico.

Respecto a los Objetivos ambientales todas las masas de agua cuentan con el objetivo específico de Buen estado al 2021, (no se han establecido Objetivos menos rigurosos).

d) Zonas protegidas y Red Natura 2000

La Memoria analiza la relación entre los espacios de la Red Natura 2000 y las zonas inundables. Así, recuerda que la Directiva 2007/60 subordina las medidas planteadas en los planes de gestión del riesgo de inundación a la obligación del cumplimiento de los objetivos ambientales definidos por los planes hidrológicos. En este mismo sentido, la Directiva Marco del Agua, a través de la designación de zonas protegidas, establece una relación directa con los objetivos de protección y conservación exigidos en otras directivas europeas como la Directiva Hábitats en relación con los espacios de la Red Natura 2000.

Pues bien, en ese marco, la Directiva de Inundaciones impulsa fundamentalmente la prevención de riesgos y la aplicación de medidas de protección del DPH, es decir, actuaciones que redundan en una disminución de los daños que causan las inundaciones y, al mismo tiempo, contribuyen al buen estado de las masas de agua a través de la mejora de sus condiciones hidromorfológicas.

Esto significa que "el plan de gestión del riesgo de inundación debe respetar la consecución del buen estado de las aguas y que este estado no se degrade en ningún caso, respetando, además, los criterios recogidos en las diversas estrategias ambientales europeas en vigor, como por ejemplo la Estrategia sobre la biodiversidad hasta 2020: nuestro seguro de vida y capital natural o la Estrategia Infraestructura verde: mejora del capital natural de Europa, siendo la Red Natura 2000 la piedra angular de la política de biodiversidad de la Unión". 
Todo ello conlleva que las zonas protegidas definidas en la Demarcación relacionadas con masas de agua deben tender a lograr el cumplimiento de todas las normas de protección que resulten aplicables en cada zona y alcanzar los objetivos ambientales particulares que en ellas se determinen.

Dando un paso más, se relaciona ahora cada ARPSI con las zonas protegidas en virtud de la Directiva 92/43/CE, indicando si el espacio protegido Red Natura 2000 lo es por sus valores como ecosistema y qué objetivos recoge para ellas el Plan Hidrológico, que serán los objetivos que establezcan los correspondientes planes de gestión de los espacios Red Natura 2000, elaborados y aprobados por las administraciones competentes.

\subsection{Planes de Protección Civil vigentes}

Respecto los Planes de protección Civil que afectan a la demarcación hidrográfica de La Gomera, se estructura esta información en tres niveles: Estatal, Autonómico y Local.

Respecto de los planes estatales, la Ley 17/2015, de 9 de julio, del Sistema Nacional de Protección Civil, establece, en su artículo 1, la protección civil como un instrumento de la política de seguridad pública, siendo el servicio público que protege a las personas y bienes garantizando una respuesta adecuada antes los distintos tipos de emergencia y catástrofes

En consecuencia, el Consejo de Ministros celebrado el 9 de diciembre de 1994 aprobó la Directriz Básica de Planificación de Protección Civil ante el Riesgo de Inundaciones. En este documento se clasifican las áreas inundables del territorio con arreglo a los criterios siguientes:

- Zona de inundación frecuente: las zonas inundables por avenidas de período de retorno de cincuenta años.

- Zonas de inundación ocasional: aquellas inundables por avenidas de período de retorno entre cincuenta y cien años.

- Zonas de inundación excepcional: las que se inundan por avenidas de período de retorno entre cien y quinientos años.

Tal y como establece la Directriz Básica de Planificación de Protección Civil ante el Riesgo de Inundaciones, a los efectos del Plan Estatal de Protección Civil ante 
el riesgo de inundaciones se considerarán todas aquellas inundaciones que representen un riesgo para la población y sus bienes, produzcan daños en infraestructuras básicas o interrumpan servicios esenciales para la comunidad, las cuales se pueden encuadrar en los tipos siguientes: (se ha de destacar que la identificación del riesgo de inundaciones se efectuará de conformidad con lo establecido en el Real Decreto 903/2010)

a) Inundaciones por precipitación «in situ».

b) Inundaciones por escorrentía, avenida o desbordamiento de cauces, provocada o potenciada por: precipitaciones, deshielo o fusión de nieve, obstrucción de cauces naturales o artificiales, invasión de cauces, aterramientos o dificultad de avenamiento y acción de las mareas.

c) Inundaciones por rotura o la operación incorrecta de obras de infraestructura hidráulica.

Respecto de los Planes Autonómicos, el artículo 3.4 de la Directriz Básica de Planificación de Protección Civil ante el Riesgo de Inundaciones establece que las Comunidades Autónomas desarrollarán unos Planes ante el Riesgo de Inundaciones en los que se definan la organización y procedimientos de actuación de los recursos y servicios cuya titularidad corresponda a la Comunidad Autónoma de que se trate y los que puedan ser asignados al mismo por otras Administraciones Públicas y de otros pertenecientes a entidades públicas o privadas, al objeto de hacer frente a las emergencias por riesgo de inundaciones, dentro del ámbito territorial de aquella.

En materia de gestión de emergencias, Canarias dispone del Plan Territorial de Emergencias de Protección Civil de la Comunidad Autónoma de Canarias, en adelante PLATECA, aprobado por el Gobierno en sesión celebrada el 12 de noviembre de 1997, siendo homologado por la Comisión Nacional de Protección Civil el 19 de diciembre de ese mismo año. Posteriormente, mediante Decreto 1/2005, de 18 de enero, el Gobierno de Canarias, aprobó la actualización del citado Plan, que fue homologada por la Comisión Nacional de Protección Civil el 28 de abril de 2005 y publicada en el BOC no 154, de 8 de agosto de 2005. Actualmente está vigente la revisión aprobada por el DECRETO 98/2015, de 22 de mayo, por el que se aprueba el Plan Territorial de Emergencias de Protección Civil de la Comunidad Autónoma de Canarias, homologada por la Comisión 
Nacional de Protección Civil el 8 de mayo de 2014 y publicada en el BOC n0104 de 2 de junio de 2015.

En virtud del artículo 3.2 del R. D. 407/1992, el PLATECA tiene el carácter de Plan Director, estableciendo el marco organizativo general para que puedan integrarse todos aquellos planes territoriales de ámbito inferior al autonómico (insulares y locales) y todos aquellos planes especiales o sectoriales cuyo ámbito sea la Comunidad Autónoma de Canarias.

El Gobierno de Canarias es competente para elaborar, aprobar y modificar el Plan Territorial de Emergencias de Protección Civil de la Comunidad Autónoma, PLATECA.

Actualmente el Plan Especial de Protección Civil ante el Riesgo de Inundaciones de Canarias (en adelante PEINCA) está elaborado, y fue informado favorablemente por la Comisión Autonómica de Protección Civil de Canarias, con fecha 24 de abril de 2017. Asimismo, la Comisión Permanente del Consejo Nacional de Protección Civil acordó informar favorablemente dicho Plan el día 12 de diciembre de 2017, conforme a lo establecido en el artículo 14.3 de la Ley 17/2015, de 9 de julio, del Sistema Nacional de Protección Civil. Posteriormente, mediante Decreto 115/2018, de 30 de julio, el Consejo de Gobierno aprobó el citado Plan, lo que fue publicado en el BOC $n^{\circ} 157$, de 14 de agosto de 2018.

El principal objetivo del PEINCA es establecer el marco organizativo en el ámbito de la Protección Civil de la Comunidad Autónoma de Canarias, mediante el cual se dé una respuesta rápida y eficaz frente a una inundación.

Finalmente, los Planes de Actuación de Ámbito Local, vienen recogidos en la Directriz Básica de Planificación de Protección Civil ante el Riesgo de Inundaciones, el Plan de cada Comunidad Autónoma debe establecer, dentro de su respectivo ámbito territorial, directrices para la elaboración de Planes de Actuación de Ámbito Local. También debe especificar el marco organizativo general que posibilite la plena integración operativa de éstos en la organización del Plan Autonómico.

Las funciones básicas de los Planes de Actuación de Ámbito Local son las siguientes: 
- Prever la estructura organizativa y los procedimientos para la intervención en emergencias por inundaciones, dentro del territorio del municipio o entidad local que corresponda.

- Catalogar elementos vulnerables y zonificar el territorio en función del riesgo, en concordancia con lo que establezca el correspondiente Plan Autonómico, así como delimitar áreas según posibles requerimientos de intervención o actuaciones para la protección de personas y bienes

- Especificar procedimientos de información y alerta a la población.

- Catalogar los medios y recursos específicos para la puesta en práctica de las actividades previstas.

Los Planes de Actuación Municipal e Insular serán aprobados por los órganos de las respectivas corporaciones locales y serán homologados por la Comisión Autonómica de Protección Civil de Canarias.

Todas las islas de la Comunidad Autónoma de Canarias deben prestar el servicio de protección civil a través de la aprobación y la efectiva implantación de su respectivo Plan de Emergencia Insular (PEIN).

Planes Fecha de Información, Homologación o Publicación

PEIN de Tenerife 17/12/2004 (se informa de su homologación

tácita el 26/07/2006)

PEIN de La Gomera 26/02/2003

PEIN de El Hierro 26 26/02/2003

PEIN de La Palma $\quad 15 / 12 / 2003$

PEIN de Gran Canaria 13/07/2006

PEIN de Lanzarote $\quad$ 09/05/2003

PEIN de Fuerteventura $\quad$ 09/05/2003

Los Planes Municipales de Protección Civil y Emergencias informados/homologados por la Comisión Autonómica de Protección Civil y atención de emergencias en la Demarcación Hidrográfica de La Gomera son: 
- PEMU de Vallehermoso, Homologado en la comisión de PC del 1 de julio de 2014

- PEMU de Alajeró, Homologado en la comisión de PC en octubre de 2015.

- PEMU de San Sebastián de La Gomera, Homologado en la comisión de PC del 24 de enero de 2017

- PEMU de Agulo, Homologado en la comisión de PC del 30 de enero de 2018.

\subsection{Plan de Gestión del Riesgo de Inundación.}

El contenido esencial del plan de gestión del riesgo de inundación es el programa de medidas. Este programa de medidas está orientado, como se recoge en el artículo 11.5 del Real Decreto 903/2010, a lograr los objetivos de la gestión del riesgo de inundación para cada zona identificada en la evaluación preliminar del riesgo de la Demarcación, partiendo de los siguientes principios generales:

a) Solidaridad: las medidas de protección contra las inundaciones no deben afectar negativamente a otras demarcaciones hidrográficas o a la parte no española de las demarcaciones hidrográficas internacionales.

b) Coordinación entre las distintas Administraciones Públicas e instituciones implicadas en materias relacionadas con las inundaciones, a partir de una clara delimitación de los objetivos respectivos.

c) Coordinación con otras políticas sectoriales, entre otras, ordenación del territorio, protección civil, agricultura, forestal, minas, urbanismo o medio ambiente, siempre que afecten a la evaluación, prevención y gestión de las inundaciones.

d) Respeto al medio ambiente: evitando el deterioro injustificado de los ecosistemas fluviales y costeros, y potenciando las medidas de tipo no estructural contra las inundaciones.

e) Planteamiento estratégico con criterios de sostenibilidad a largo plazo.

f) Los programas de medidas son el conjunto de actuaciones a llevar a cabo por la administración competente en cada caso. Los planes de gestión del riesgo de inundación deben tener en cuenta aspectos pertinentes tales como los costes y beneficios, la extensión de la inundación y las vías de evacuación de inundaciones, las zonas con potencial de retención de las 
inundaciones, las llanuras aluviales naturales, los objetivos medioambientales indicados en el artículo 92 bis del Real Decreto Legislativo 1/2001, de 20 de julio, por el que se aprueba el Texto Refundido de la Ley de Aguas, la gestión del suelo y del agua, la ordenación del territorio, el uso del suelo, la conservación de la naturaleza, la navegación e infraestructuras de puertos.

De acuerdo con el punto artículo 11.4 del Real Decreto 903/2010, los planes de gestión del riesgo de inundación deben abarcar todos los aspectos de la gestión del riesgo de inundación, centrándose en la prevención, protección y preparación, incluidos la previsión de inundaciones y los sistemas de alerta temprana, y teniendo en cuenta las características de la cuenca o subcuenca hidrográfica considerada. En la Parte A: Contenido de los planes de gestión del riesgo de inundación del Anexo del Real Decreto 903/2010, se recogen los tipos de medidas que, en lo posible, deberán contemplar los programas de medidas. Por otro lado, la Comisión Europea en el documento Guidance Document No.29 Guidance for Reporting under the Floods Directive, describe los tipos de medidas en función del aspecto de la gestión del riesgo sobre el que actúan.

Teniendo esto en cuenta, a continuación, se detallan las medidas incluidas en el plan de gestión del riesgo de inundación, clasificadas según las siguientes categorías:

- Medidas de restauración fluvial y medidas para la restauración hidrológico-agroforestal.

- Medidas de mejora del drenaje de infraestructuras lineales.

- Medidas de predicción de avenidas

- Medidas de protección civil.

- Medidas de ordenación territorial y urbanismo.

- Medidas para promocionar los seguros.

- Medidas estructurales y estudios coste-beneficio que las justifican.

Comentario de las medidas más relevantes.

En relación con la Ordenación del territorio y el urbanismo, las medidas relacionadas con la ordenación territorial y el urbanismo están recogidas en el punto 5 del apartado I.h) de la parte A del Anexo del Real Decreto 903/2010, de 
9 de julio, de evaluación y gestión de riesgos de inundación, y según establece esta disposición, incluirán al menos:

"Las limitaciones a los usos de suelo planteadas para la zona inundable en sus diferentes escenarios de peligrosidad, los criterios empleados para considerar el territorio como no urbanizable, y los criterios constructivos exigidos a las edificaciones situadas en zona inundable.

Las medidas para adaptar el planeamiento urbanístico vigente a los criterios planteados en el plan de gestión del riesgo de inundación incluida la posibilidad de retirar construcciones existentes que supongan un grave riesgo, para lo cual su expropiación tendrá la consideración de utilidad pública."

Normativa general.

En esta materia es de aplicación, en primer término, la Ley del Suelo y Rehabilitación Urbana, Real Decreto Legislativo 7/2015, de 30 de diciembre, por el que se aprueba el Texto refundido de la Ley del Suelo y Rehabilitación Urbana (en adelante TRLSRU), y a las diferentes leyes de suelo y ordenación del territorio autonómicas, junto con la normativa de desarrollo, que en todo caso habrá de estar a los planes de ordenación urbana de los municipios. En la Comunidad Autónoma de Canarias, ha de tenerse en cuenta la Ley 4/2017, de 13 de julio, del Suelo y de los Espacios Naturales Protegidos de Canarias (en adelante Ley del Suelo de Canarias).

De conformidad con el artículo 21 del TRLSRU, todo el suelo se encuentra en una de las situaciones básicas de suelo rural o de suelo urbanizado, y está en la situación de suelo rural: "En todo caso, el suelo preservado por la ordenación territorial y urbanística de su transformación mediante la urbanización, que deberá incluir, como mínimo, los terrenos excluidos de dicha transformación por la legislación de protección o policía del dominio público, de la naturaleza o del patrimonio cultural, los que deban quedar sujetos a tal protección conforme a la ordenación territorial y urbanística por los valores en ellos concurrentes, incluso los ecológicos, agrícolas, ganaderos, forestales y paisajísticos, así como aquéllos con riesgos naturales o tecnológicos, incluidos los de inundación o de otros accidentes graves, y cuantos otros prevea la legislación de ordenación territorial o urbanística". 
En cuanto a la normativa autonómica de Canarias, ha de tenerse en cuenta lo dispuesto en el artículo 33 de la Ley del Suelo de Canarias, se incluye la siguiente definición de suelo rústico:

1. El suelo rústico (SR) está integrado por los terrenos que el planeamiento adscriba a esta clase de suelo por concurrir en ellos alguna de las siguientes características:

“e) Ser necesaria su protección por riesgos ciertos naturales o tecnológicos, incluidos los de inundación, erosión, desprendimientos, corrimientos o fenómenos análogos que comporten sus características geotécnicas o morfológicas".

Y en el artículo 34 de la Ley del Suelo de Canarias, se establecen las siguientes categorías de suelo rústico si bien el propio plan destaca que no existe ninguna categoría específica que englobe a los suelos con riesgos, como sería el caso de los suelos inundables.

En todo caso, en esta materia se2 destaca la coordinación y así se recuerda que En materia de gestión de zonas inundables es muy importante la labor de coordinación de los Consejos Insulares de Agua con las administraciones competentes en materia de urbanismo, así como las limitaciones de uso que mediante decreto puede establecer el Gobierno de Canarias. El artículo 16 del Decreto 86/2002, de 2 de julio, por el que se aprueba el Reglamento de Dominio Público Hidráulico, establece que:

"16.1 Se podrán considerar como zonas anegables las cubiertas por las aguas de las avenidas con periodo estimado de recurrencia no superior a quinientos años.

16.2 Mediante decreto, el Gobierno de Canarias podrá establecer las limitaciones en el uso de las zonas anegables que se estimen necesarias para garantizar la seguridad de personas y bienes".

Además, se recuerda en cuanto a usos permitidos, el Reglamento del Dominio Público Hidráulico establece en su artículo 9 que en las zonas o vías de flujo preferente solo podrán ser autorizadas por el organismo de cuenca aquellas 
actividades no vulnerables frente a avenidas y que no supongan una reducción significativa de la capacidad de desagüe de dicha vía.

Del mismo modo, respecto al ámbito costero, la Ley 22/1988, de 28 de julio, de Costas, incluye en el art. 3 (y concordantes del Real Decreto 876/2014, de 10 de octubre, por el que se aprueba el Reglamento General de Costas), la clasificación de los bienes de dominio público marítimo-terrestre estatal, entre los que se incluyen el mar territorial y las aguas interiores, con su lecho y subsuelo y los recursos naturales de la zona económica y la plataforma continental, y establece las limitaciones y servidumbres a las que están sujetos los terrenos colindantes con el dominio público marítimo-terrestre. La Ley de Costas distingue entre zona de servidumbre de protección, zona de servidumbre de tránsito y zona de servidumbre de acceso al mar (art. 23-28 de la Ley y concordantes del Reglamento). Así como detallan las limitaciones al desarrollo urbanístico para las zonas de servidumbre de protección y servidumbre de tránsito.

Normativa específica.

En cuanto a la normativa específica se hace referencia, a la normativa específica en materia de aguas.

Así, resulta de aplicación el Plan hidrológico (ciclo 2015-2021) y Plan de Gestión de Riesgo de Inundación La Ley de Aguas de Canarias, determina en su artículo 38, que los Planes Hidrológicos Insulares comprenderán entre otros muchos aspectos, los criterios sobre estudios, actuaciones y obras para prevenir y evitar los daños debidos a inundaciones, avenidas y otros fenómenos hidráulicos.

En el caso de la Demarcación Hidrográfica de La Gomera, el Plan Hidrológico vigente se corresponde con el de segundo ciclo (2015-2021), aprobado definitivamente mediante Decreto 137/2018, de 17 de septiembre. El Plan Hidrológico Insular vigente regula lo siguiente:

Artículo 35. Estudios hidrológicos e hidráulicos previos a la autorización de obras en cauces:

1. Los promotores que pretendan ejecutar obras en los cauces o en las zonas anexas a los mismos sujetas legalmente a algún tipo de limitación en su 
uso, deberán presentar con carácter previo al otorgamiento del correspondiente título administrativo los estudios hidrológicos e hidráulicos empleados para su definición.

2. Salvo justificación en contra, que debe ser aceptada expresamente por el Consejo Insular de Aguas de La Gomera, el estudio hidráulico deberá realizarse mediante modelación, preferiblemente del régimen variado, por medio de herramientas informáticas que integren la metodología Standard Step Method, utilizando una combinación de las ecuaciones de energía, momento y continuidad.

Artículo 36. Norma generales en relación con los cauces y márgenes:

1. De acuerdo con el artículo 60 de la Ley de Aguas de Canarias el Consejo Insular de Aguas de La Gomera, de oficio o a instancia de parte, procederá a efectuar el deslinde de aquellos cauces en que se prevean o aprecien acciones capaces de proyectarse sobre el cauce o su zona de servidumbre y, en su caso, ejercerá la potestad de recuperación de oficio para preservar la integridad del dominio público hidráulico superficial.

2. El deslinde de los cauces y la delimitación de sus zonas de servidumbre y policía se realizarán atendiendo a los mandatos establecidos en el artículo 8 y siguientes del Reglamento de Dominio Público Hidráulico, aprobado por Decreto $86 / 2002$, de 2 de julio.

3. Las restricciones a los usos en las zonas de policía y de servidumbre serán como mínimo las incluidas en los artículos 12 y 13 respectivamente del Reglamento de Dominio Público Hidráulico.

Artículo 37. Protección contra inundaciones (ND):

1. Conforme a lo establecido en el Real Decreto 903/2010 de 9 de julio, de evaluación y gestión de riesgos de inundación, el Consejo Insular de Aguas de La Gomera realizará la evaluación preliminar del riesgo de inundación, los mapas de peligrosidad y riesgo y el plan de gestión de los riesgos de inundación.

2. Las distintas Administraciones públicas, dentro de sus respectivas competencias, elaborarán los programas de medidas y desarrollarán las 
actuaciones derivadas de los mismos en el ámbito de los planes de gestión del riesgo de inundación, impulsando la coordinación entre sus organismos.

Analizada la normativa, en cuanto a los objetivos del Plan de gestión que cubre esta medida. Así se afirma que un aspecto fundamental en la gestión de riesgo de inundación es la ordenación hidráulica de las llanuras inundables, que debe conseguir una adecuada compatibilidad de usos que evite que los nuevos desarrollos urbanos conlleven un aumento de riesgo a futuro. Si bien la Directiva Europea de Inundaciones se centra sobre todo en la reducción de los riesgos actuales, no tendría sentido realizar actuaciones con este objetivo si en paralelo se autorizan usos vulnerables en zonas con peligrosidad elevada.

Esta medida es, por tanto, esencial para alcanzar el objetivo de contribuir a mejorar la ordenación del territorio y la gestión de la exposición en las zonas inundables. Se basa en la búsqueda de las mejores opciones medioambientalmente posibles que favorezcan usos del suelo compatibles con las inundaciones, todo ello conforme a la legislación vigente en materia de suelo y urbanismo, protección civil, costas, aguas, medio ambiente, etc., y mejorando la consideración de las inundaciones en los distintos instrumentos de ordenación del territorio.

Además de este objetivo básico, esta medida ayuda notablemente a la consecución de otros objetivos incluidos en el Plan de Gestión del Riesgo de Inundación, como son los siguientes:

La ordenación del territorio es quizá el enfoque más eficaz para prevenir el incremento en el riesgo de inundación, o en su caso reducirlo, de una forma sostenible, mediante el control de los usos y el establecimiento de criterios para el desarrollo de las distintas actividades en las zonas potencialmente inundables.

En cuanto a las competencias, corresponde a la comunidad autónoma de Canarias, ejercer las competencias que ejercen los Consejos Insulares de Aguas.

El segundo aspecto importante es el relativo al Urbanismo, y más concretamente las medidas previstas para adaptar el planeamiento urbanístico 
Los objetivos del Plan de gestión que cubre esta medida se refieren a la mejorar de la resiliencia y disminuir la vulnerabilidad de los elementos ubicados en las zonas inundables. Puesto que las inundaciones son fenómenos naturales que no pueden evitarse y hay que convivir con ellas asumiendo un cierto nivel de riesgo, más aún con los previsibles efectos del cambio climático, se prevé la necesidad de adaptar progresivamente los bienes, tales como viviendas, infraestructuras, etc. existentes en las zonas inundables para que los daños que se produzcan en una eventual inundación sean lo menores posibles.

Además de este objetivo básico, esta medida ayuda notablemente a la consecución de otros objetivos incluidos en el Plan de Gestión del Riesgo de Inundación, como son los siguientes:

- Contribuir a mejorar la ordenación del territorio y la gestión de la exposición en las zonas inundables. Se basa en la búsqueda de las mejores opciones medioambientalmente posibles que favorezcan usos del suelo compatibles con las inundaciones, todo ello conforme a la legislación vigente en materia de suelo y urbanismo, protección civil, costas, aguas, medio ambiente, etc., y mejorando la consideración de las inundaciones en los distintos instrumentos de ordenación del territorio.

- Incremento de la percepción del riesgo de inundación y de las estrategias de autoprotección en la población, los agentes sociales y económicos, puesto que la integración de los criterios de protección frente a inundaciones en los instrumentos de ordenación del territorio y planeamiento urbanístico, junto con la correspondiente cartografía de inundabilidad, son herramientas de concienciación de la sociedad ante el fenómeno de la inundación.

- Mejorar la coordinación administrativa entre todos los actores involucrados en la gestión del riesgo, mediante el intercambio de información y la promoción de actividades de formación y concienciación.

- Conseguir una reducción, en la medida de lo posible, del riesgo a través de la disminución de la peligrosidad para la salud humana, las actividades económicas, el patrimonio cultural y el medio ambiente en las zonas inundables ya que una adecuada gestión de los usos en la zona inundable proporciona, por un lado, las condiciones para que las llanuras aluviales 
puedan ejercer su función en la laminación de avenidas, para el aumento de la capacidad de retención de agua en el suelo, etc., y por otro, el espacio necesario para la ejecución de medidas de protección.

Para alcanzar estos objetivos, se ha detectado la necesidad de adaptar el planeamiento urbanístico a las determinaciones y criterios de los planes de gestión del riesgo de inundación, e impulsar la revisión de planes generales municipales en ARPSIs, en concreto se llevará a cabo esta medida en los siguientes municipios:

- ARPSI_005 Barranco Playa de Santiago, Alajeró, Plan general, 2005

- ARPSI_006 Barranco de Valle Gran rey, Valle Gran Rey, Normas subsidiarias, 1989.

- ARPSI_007 Barranco de San Sebastián, San Sebastián de La Gomera, Plan general 2006.

Los Planes Generales de Ordenación Urbana o Normas Subsidiarias, además de incluir la delimitación de zonas inundables y los usos permitidos, deben adaptar las ordenanzas en cada caso, tomando, a modo de ejemplo, medidas como las siguientes:

- Adopción de dispositivos diseñados para la protección contra inundaciones de propiedades. Estos incluyen barreras desmontables diseñadas para adaptarse a aberturas, tapas de ventilación y tapones, etc.

- Elevación de los niveles de umbral y el piso por encima de los niveles de inundación esperados. Esto se logra típicamente elevando altura de piso dentro de la estructura del edificio.

- Otras recomendaciones con respecto a la orientación de la edificación frente al flujo o al nivel pueden ser de interés para reducir la peligrosidad de la avenida.

- Medidas para mejorar la resiliencia de la edificación (entendiendo estas como la capacidad para recuperarse después de la inundación) de tal manera que la recuperación post-inundación sea más rápida.

- Medidas para la ordenación de usos dentro de la edificación.

- Otro aspecto destacable es la importancia que el transporte, y con ello la viabilidad, ha ido tomando en las sociedades modernas y que adquiere 
un singular relieve en situaciones de inundación por las interrupciones masivas que pueden llegar a producirse. La incomunicación viaria puede llegar a impedir la evacuación y la llegada de ayuda de emergencias, lo que puede desembocar en problemas a la hora de atender a zonas que puedan verse afectadas por inundaciones y que puede provocar la imposibilidad de rescate de ciudadanos que se encuentren en zonas susceptibles. Por ello, la situación de la red viaria en las zonas inundables se convierte en un elemento clave de la política de defensa contra inundaciones y que habrá de ser analizada minuciosamente también en la redacción del planeamiento urbanístico.

Otras medidas se refieren a los siguientes aspectos.

- Reordenación de los usos del suelo en las zonas inundables haciéndolos compatibles con las inundaciones (relocalización o retirada de actividades/instalaciones vulnerables) (13.02.01)

- Adaptación de elementos en zonas inundables para reducir las consecuencias adversas en episodios de inundaciones en viviendas, edificios públicos, redes, etc.

- $\quad$ Elaboración de estudios de mejora del conocimiento sobre la gestión del riesgo de inundación

- $\quad$ Programa de mantenimiento y conservación de cauces

- $\quad$ Programa de mantenimiento y conservación del litoral

- Medidas en la cuenca: restauración hidrológico-forestal y ordenaciones agrohidrológicas

- Medidas en cauce y llanura de inundación: restauración fluvial, incluyendo medidas de retención natural y reforestación de riberas

- $\quad$ Medidas de restauración de la franja costera y la ribera del mar

- $\quad$ Normas de gestión de la explotación de embalses durante las avenidas

- Medidas estructurales para regular los caudales, tales como la construcción y/o modificación de presas 
- $\quad$ Mejora del drenaje de infraestructuras lineales: carreteras, ferrocarriles

- $\quad$ Medidas estructurales (encauzamientos, motas, diques, etc.) que implican intervenciones físicas en los cauces, aguas costeras y áreas propensas a inundaciones

- $\quad$ Medidas que implican intervenciones físicas para reducir las inundaciones por aguas superficiales, por lo general, en un entorno urbano, como la mejora de la capacidad de drenaje artificial o sistemas de drenaje sostenible (SUDS)

- Establecimiento y mejora de los sistemas de alerta meteorológica incluyendo los sistemas de medida y predicción de temporales marinos

- Establecimiento y mejora los sistemas de medida y alerta hidrológica

- Medidas para establecer o mejorar la planificación institucional de respuesta a emergencias de inundaciones a través de la coordinación con planes de protección civil

- Mejora de los protocolos de actuación y comunicación de la información relativa a inundaciones

- Medidas para establecer o mejorar la conciencia pública en la preparación para las inundaciones, para incrementar la percepción del riesgo de inundación y de las estrategias de autoprotección en la población, los agentes sociales y económicos

- Obras de emergencia para reparación de infraestructuras afectadas, incluyendo infraestructuras sanitarias y ambientales básicas

- $\quad$ Planes de protección civil: acciones de apoyo a la salud, asistencia financiera, incluida asistencia legal, así como reubicación temporal de la población afectada

- Promoción del seguro sobre personas y bienes, especialmente los seguros agrarios

- Evaluación, análisis y diagnóstico de las lecciones aprendidas en la gestión de los eventos de inundación

Y para cada uno de ellos, se tratan los siguientes aspectos: 
- Introducción

- $\quad$ Objetivos del Plan de gestión que cubre esta medida

- Descripción de la medida / Actuaciones específicas a llevar a cabo

- $\quad$ Organismos responsables de la implantación

- $\quad$ Costes y beneficios generales de la medida

- Aspectos claves en el calendario de su implantación y descripción del grado de avance 